\title{
Dispersionless Hirota Equations and the Genus 3 Hyperelliptic Divisor
}

\author{
Fabien Cléry, Evgeny V. FerapontoviD \\ Department of Mathematical Sciences, Loughborough University, Loughborough, UK \\ E-mail: cleryfabien@gmail.com; E.V.Ferapontov@1boro.ac.uk
}

Received: 26 March 2019 / Accepted: 8 July 2019

Published online: 19 August 2019 - (C) The Author(s) 2019

Dedicated to the memory of Professor Boris Dubrovin

Abstract: Equations of dispersionless Hirota type

$$
F\left(u_{x_{i} x_{j}}\right)=0
$$

have been thoroughly investigated in mathematical physics and differential geometry. It is known that the parameter space of integrable Hirota type equations in $3 \mathrm{D}$ is 21 dimensional, and that the action of the natural equivalence group $\operatorname{Sp}(6, \mathbb{R})$ on the parameter space has an open orbit. However the structure of the generic equation corresponding to the open orbit remained elusive. Here we prove that the generic 3D Hirota equation is given by the remarkable formula

$$
\vartheta_{m}(\tau)=0, \quad \tau=i \operatorname{Hess}(u)
$$

where $\vartheta_{m}$ is any genus 3 theta constant with even characteristics and $\operatorname{Hess}(u)$ is the $3 \times 3$ Hessian matrix of a (real-valued) function $u\left(x_{1}, x_{2}, x_{3}\right)$. Thus, generic Hirota equation coincides with the equation of the genus 3 hyperelliptic divisor (to be precise, its intersection with the imaginary part of the Siegel upper half space $\mathfrak{H}_{3}$ ). The rich geometry of integrable Hirota type equations sheds new light on local differential geometry of the genus 3 hyperelliptic divisor, in particular, the integrability conditions can be viewed as local differential-geometric constraints that characterise the hyperelliptic divisor uniquely modulo $\operatorname{Sp}(6, \mathbb{C})$-equivalence.

\section{Introduction}

A 3D dispersionless Hirota type equation is a second-order PDE of the form

$$
F\left(u_{x_{i} x_{j}}\right)=0
$$

where $u$ is a function of 3 independent variables. Equations of type (1.1) arise in numerous applications in non-linear physics, general relativity, differential geometry, theory 
of integrable systems, and complex analysis. For instance, dispersionless KadomtsevPetviashvili (dKP) equation

$$
u_{x_{1} x_{3}}-\frac{1}{2} u_{x_{1} x_{1}}^{2}-u_{x_{2} x_{2}}=0
$$

arises in non-linear acoustics [45], the theory of Einstein-Weyl structures [12] and in the context of conformal maps of simply-connected domains [32,44,46]. The Boyer-Finley (BF) equation

$$
u_{x_{1} x_{1}}+u_{x_{2} x_{2}}-e^{u_{x_{3} x_{3}}}=0
$$

describes a class of self-dual Ricci-flat 4-manifolds [1]. Let us review briefly some of the known properties of integrable Hirota type equations (see Sect. 2 for a summary of four equivalent approaches to dispersionless integrability in 3D):

- The class of integrable equations (1.1) is invariant under the symplectic group $\operatorname{Sp}(6, \mathbb{R})$ via its standard action

$$
U \mapsto(A U+B)(C U+D)^{-1} .
$$

Here $U=\operatorname{Hess}(u)$ is the Hessian matrix of the function $u$ [14]. The classification of integrable equations is performed modulo equivalence (1.3).

- The parameter space of integrable equations (1.1) is 21-dimensional (see theorem 1 of [14]). Furthermore, the action of the equivalence group $\operatorname{Sp}(6, \mathbb{R})$ on the parameter space is locally free. The fact that the group $\operatorname{Sp}(6, \mathbb{R})$ is 21 -dimensional implies the existence of a generic Hirota equation generating an open 21-dimensional $\operatorname{Sp}(6, \mathbb{R})$-orbit. Any representative of the open orbit will be called a Hirota master-equation. The masterequation has no continuous symmetries from the equivalence group. Note that both $\mathrm{dKP}$ and $\mathrm{BF}$ equations have non-trivial symmetry groups and generate singular orbits of lower dimensions (14 and 15, respectively).

- Geometrically, Hirota type Eq. (1.1) can be viewed as the defining equation of a 5dimensional hypersurface $M^{5}$ in the 6-dimensional Lagrangian Grassmannian $\Lambda^{6}$ (locally identified with $3 \times 3$ symmetric matrices). Integrability of Eq. (1.1) can be interpreted as integrability of a $\operatorname{GL}(2, \mathbb{R})$ geometry induced on $M^{5}$ (see [42] and Section 4 of [14]).

Although the structure of the Hirota master-equation has remained elusive, formula (1.3) suggests that this equation should possess non-trivial modular properties. In this direction let us mention yet another integrable example,

$$
u_{x_{3} x_{3}}-\frac{u_{x_{1} x_{2}}}{u_{x_{1} x_{3}}}-\frac{1}{6} h\left(u_{x_{1} x_{1}}\right) u_{x_{1} x_{3}}^{2}=0
$$

which was discussed in $[37,38]$. Here the function $h$ satisfies the Chazy equation

$$
h^{\prime \prime \prime}+2 h h^{\prime \prime}-3\left(h^{\prime}\right)^{2}=0
$$

whose general solution can be expressed in terms of the Eisenstein series of weight 2 on the modular group $\operatorname{SL}(2, \mathbb{Z}): h(t)=e_{2}(i t / \pi)$ where

$e_{2}(\tau)=1-24 \sum_{n=1}^{\infty} \sigma_{1}(n) e^{2 \pi i n \tau}=1-24 q-72 q^{2}-96 q^{3}+\cdots \quad$ where $q=e^{2 \pi i \tau}$. 
Note that $h(t)$ is real for $t>0$. This was one of the first examples where modular forms explicitly occurred as coefficients rather than as solutions of integrable systems, see also $[2,13,31]$; note that PDE (1.4) admits non-trivial continuous symmetries and therefore does not generate an open orbit. We refer to Sect. 4 for further 'modular' examples.

Before giving our main theorem, let us recall few facts about theta constants. All these facts can be found in [29]. Let $\mathfrak{H}_{3}$ be the Siegel upper half space of genus 3 i.e.

$$
\mathfrak{H}_{3}=\left\{\tau \in \operatorname{Mat}(3 \times 3, \mathbb{C}) \mid \tau=\tau^{t}, \operatorname{Im}(\tau)>0\right\} .
$$

Theta constants with characteristics are defined by

$$
\vartheta_{m}(\tau)=\vartheta_{\left[\begin{array}{l}
\mu \\
v
\end{array}\right]}(\tau)=\sum_{n \in \mathbb{Z}^{3}} e^{i \pi(n+\mu / 2)\left(\tau(n+\mu / 2)^{t}+v^{t}\right)}
$$

where $\mu, v \in\{0,1\}^{3}$ and $\tau \in \mathfrak{H}_{3}$. The characteristic $m=\left[\begin{array}{l}\mu \\ v\end{array}\right]$ is called even if $\mu v^{t}$ is even. In genus 3 , there are 36 such characteristics and they give rise to 36 theta constants which are modular forms of weight one-half with a miltiplier system (see [23], theorem 1.1) on the so-called Igusa group $\Gamma_{3}(4,8)$ defined as

$$
\begin{aligned}
\Gamma_{3}(4,8)= & \left\{M=\left(\begin{array}{ll}
a & b \\
c & d
\end{array}\right) \in \operatorname{Sp}(6, \mathbb{Z}): M \equiv I_{6} \bmod 4, \operatorname{diag}\left(a^{t} b\right)\right. \\
& \left.\equiv \operatorname{diag}\left(c^{t} d\right) \equiv 0 \bmod 8\right\} .
\end{aligned}
$$

Recall that there is an action of the group $\operatorname{Sp}(6, \mathbb{Z})$ on the set of even characteristics which is transitive. The product of 36 theta constants with even characteristics equals a cusp form of weight 18 on the full modular group $\operatorname{Sp}(6, \mathbb{Z})$, this form is classically denoted by $\chi_{18}$ (see [28]). It is known that in genus 3 the vanishing of even theta constants characterises the hyperelliptic divisor which consists of 36 irreducible isomorphic components. Note that $\vartheta_{m}(\tau)$ is real when $\tau$ is purely imaginary.

Our main result is the following explicit formula for the master-equation:

Theorem 1.1. The master-equation is given by the formula

$$
\vartheta_{m}(\tau)=0, \quad \tau=i \operatorname{Hess}(u)
$$

where $\vartheta_{m}$ is any genus 3 theta constant with even characteristic $m$ and Hess $(u)$ is the Hessian matrix of the function $u$. Equation (1.6) defines a hypersurface $M^{5}$ in the Lagrangian Grassmannian $\Lambda^{6}$ (we assume Hess $(u)$ to be positive definite). Embedding the positive cone of $\Lambda^{6}$ into the purely imaginary locus of the Siegel upper half space $\mathfrak{H}_{3}$ via multiplication by $i$, we can thus characterise $M^{5}$ as the intersection of the purely imaginary locus with the genus 3 hyperelliptic divisor.

Remark 1.2. Note that although there are 36 even theta constants in genus 3 , the corresponding equations (1.6) are all equivalent (over $\mathbb{C}$ ) due to the transitivity of the $\operatorname{Sp}(6, \mathbb{Z})$-action on the set of even theta constants. Using the cusp form $\chi_{18}$, the equations (1.6) can be compactly represented as

$$
\chi_{18}(i \operatorname{Hess}(u))=0 .
$$

Theorem 1.1 will be proved in Sect. 3 by uncovering geometry behind the OdesskiiSokolov construction [36] that parametrises broad classes of dispersionless integrable systems via generalised hypergeometric functions.

There exist several approaches to dispersionless integrability in 3D. Based on seemingly different ideas they however lead to equivalent classification results. These approaches are: 
- The method of hydrodynamic reductions based on the requirement that Eq. (1.1) has 'sufficiently many' special multiphase solutions.

- Integrability on equation, meaning that the associated hypersurface $M^{5} \subset \Lambda^{6}$ carries an integrable $\mathrm{GL}(2, \mathbb{R})$ geometry.

- Integrability on solutions, based on the requirement that the characteristic variety of Eq. (1.1) defines Einstein-Weyl geometry on every solution.

- Integrability via a dispersionless Lax representation.

The above properties of the master-equation shed new light on local differential geometry of the genus 3 hyperelliptic divisor, see Sects. 2.1-2.4.

\section{Four Equivalent Approaches to Dispersionless Integrability}

In this section we summarise the existing approaches to dispersionless integrability in 3D.

2.1. Integrability via hydrodynamic reductions. The method of hydrodynamic reductions applies to quasilinear systems of the form

$$
A(\mathbf{v}) \mathbf{v}_{x_{1}}+B(\mathbf{v}) \mathbf{v}_{x_{2}}+C(\mathbf{v}) \mathbf{v}_{x_{3}}=0
$$

where $\mathbf{v}=\left(v^{1}, \ldots, v^{m}\right)^{t}$ is an $m$-component column vector of the dependent variables and $A, B, C$ are $l \times m$ matrices, $l \geq m$. Note that Hirota type Eq. (1.1) can be brought to quasilinear form (2.1) by representing it in evolutionary form,

$$
u_{x_{1} x_{1}}=f\left(u_{x_{1} x_{2}}, u_{x_{1} x_{3}}, u_{x_{2} x_{2}}, u_{x_{2} x_{3}}, u_{x_{3} x_{3}}\right),
$$

choosing the arguments of $f$ as the new dependent variables $\mathbf{v}$ and writing out all possible consistency conditions among them. This results in the quasilinear representation (2.1) with $m=5, l=8$. Applied to system (2.1) the method of hydrodynamic reductions consists of seeking multiphase solutions in the form

$$
\mathbf{v}=\mathbf{v}\left(R^{1}, \ldots, R^{N}\right)
$$

where the phases (Riemann invariants) $R^{i}\left(x_{1}, x_{2}, x_{3}\right)$, whose number $N$ is allowed to be arbitrary, are required to satisfy a pair of commuting $(1+1)$-dimensional systems

$$
R_{x_{2}}^{i}=\mu^{i}(R) R_{x_{1}}^{i}, \quad R_{x_{3}}^{i}=\lambda^{i}(R) R_{x_{1}}^{i}
$$

known as systems of hydrodynamic type $[11,43]$. The corresponding characteristic speeds $\mu^{i}, \lambda^{i}$ must satisfy the commutativity conditions [43]

$$
\frac{\partial_{j} \mu^{i}}{\mu^{j}-\mu^{i}}=\frac{\partial_{j} \lambda^{i}}{\lambda^{j}-\lambda^{i}},
$$

where $i, j \in\{1, \ldots, N\}, i \neq j, \partial_{j}=\partial_{R^{j}}$. Equations (2.3) are said to define an $N$-component hydrodynamic reduction of system (2.1). The following definition was proposed in [13], see also [22]:

Definition 2.1. System (2.1) is said to be integrable if, for every $N$, it admits infinitely many $N$-component hydrodynamic reductions parametrised by $2 N$ arbitrary functions of one variable. 
This requirement imposes strong constraints (integrability conditions) on the matrix entries of $A, B$ and $C$. Applied to equation (2.2), the method of hydrodynamic reductions leads to an $\operatorname{Sp}(6, \mathbb{C})$-invariant set of differential constraints for the function $f$ expressing all third-order partial derivatives of $f$ in terms of its first and second-order partial derivatives ( 35 relations that are rational in the partial derivatives of $f$ ). The integrability conditions were first derived in [14]. Their involutivity implies that the parameter space of integrable Hirota type equations is 21-dimensional [14].

Note that (2.2) is, locally, the equation of the graph of the corresponding hypersurface $M^{5} \subset \Lambda^{6}$. Our main result (Theorem 1.1) states that Hirota master-equation coincides with the equation of the genus 3 hyperelliptic divisor. Thus, the integrability conditions can be viewed as local differential constraints that characterise the hyperelliptic divisor uniquely modulo $\operatorname{Sp}(6, \mathbb{C})$-equivalence.

2.2. Integrability on equation: integrable $\operatorname{GL}(2, \mathbb{R})$ geometry. The Lagrangian Grassmannian $\Lambda^{6}$ (locally parametrised by $3 \times 3$ symmetric matrices) carries a flat generalised conformal structure defined by the family of degree 4 Veronese cones in $T \Lambda^{6}$ (identified with rank 1 symmetric matrices). Let $M^{5}$ be a hypersurface in $\Lambda^{6}$. Taking a point $s \in M^{5}$ and intersecting the tangent space $T_{S} M^{5}$ with the Veronese cone in $T_{S} \Lambda^{6}$ one obtains a rational normal cone of degree 4 in $T_{S} M^{5}$. On projectivisation, this results in a family of rational normal curves $\gamma$ of degree 4 in $\mathbb{P} T M^{5}$. This structure is known as $\operatorname{GL}(2, \mathbb{R})$ geometry on $M^{5}$.

Definition 2.2. A bisecant plane in $T M^{5}$ is a plane whose projectivisation is a bisecant line of $\gamma$.

A bisecant surface is a 2-dimensional submanifold $\Sigma^{2} \subset M^{5}$ whose tangent planes are bisecant.

A trisecant space in $T M^{5}$ is a 3-dimensional subspace whose projectivisation is a trisecant plane of $\gamma$.

A trisecant 3-fold is a 3-dimensional submanifold $\Sigma^{3} \subset M^{5}$ whose tangent spaces are trisecant.

To be more precise, we will need holonomic trisecant 3-folds which can be defined as follows. Note that each tangent space $T \Sigma^{3}$ carries 3 distinguished directions, namely those corresponding to the 3 points of intersection of $\mathbb{P} T \Sigma^{3}$ with $\gamma$. These directions define a net on $\Sigma^{3}$ which we require to be holonomic i.e. a coordinate net.

It turns out that bisecant surfaces and holonomic trisecant 3-folds of a hypersurface $M^{5}$ correspond to 2- and 3-component hydrodynamic reductions of the associated Hirota type equation. Furthermore, the hypersurface $M^{5}$ corresponds to an integrable equation if and only if it has infinitely many holonomic trisecant 3 -folds parametrised by 3 arbitrary functions of one variable [14]. Thus the existence of holonomic trisecant 3-folds is a geometric interpretation of the integrability property. The corresponding integrable $\operatorname{GL}(2, \mathbb{R})$ geometries were thoroughly investigated in [42].

Thus, Theorem 1.1 implies that the genus 3 hyperelliptic divisor carries integrable $\mathrm{GL}(2, \mathbb{R})$ geometry.

2.3. Integrability on solutions: Einstein-Weyl geometry. Any solution of equation (1.1) carries a family of characteristic cones

$$
\frac{\partial F}{\partial u_{x_{i} x_{j}}} p_{i} p_{j}=0,
$$


assumed to be non-degenerate. The inverse matrix of the associated quadratic form gives rise to the conformal structure $[g]=g_{i j} d x_{i} d x_{j}$ which depends on a solution due to nonlinearity of (1.1). It was shown in Theorem 7 of [17] that integrability of equation (1.1) is equivalent to the requirement that conformal structure $[g]$ is Einstein-Weyl on every solution. Recall that an Einstein-Weyl structure consists of a symmetric connection $\mathbb{D}$ and a conformal structure $[g]$ such that

- connection $\mathbb{D}$ preserves the conformal class: $\mathbb{D}[g]=0$,

- trace-free part of the symmetrised Ricci tensor of $\mathbb{D}$ vanishes.

In local coordinates,

$$
\mathbb{D}_{k} g_{i j}=\omega_{k} g_{i j}, \quad R_{(i j)}=\rho g_{i j}
$$

where $\omega=\omega_{k} d x_{k}$ is a covector, $R_{(i j)}$ is the symmetrised Ricci tensor of $\mathbb{D}$, and $\rho$ is some function. It was shown in Section 5 of [17] that the covector $\omega$ can be expressed in terms of $g$ by the universal explicit formula

$$
\omega_{k}=2 g_{k j} \mathcal{D}_{x_{s}}\left(g^{j s}\right)+\mathcal{D}_{x_{k}}\left(\ln \operatorname{det} g_{i j}\right)
$$

where $\mathcal{D}_{x_{k}}$ denotes the total derivative with respect to $x_{k}$. We recall that in $3 \mathrm{D}$ the Einstein-Weyl equations (2.4) are integrable by twistor-theoretic methods [25]. Thus solutions of integrable PDEs carry 'integrable' conformal geometry. Combining results of Sects. 2.2 and 2.3 with Theorem 1.1 we can conclude that every trisecant 3 -fold of the hyperelliptic divisor carries Einstein-Weyl geometry.

Cartan proved (see $[6])$ that a pair $(\mathbb{D},[g])$ defined on a 3-dimensional manifold satisfies Einstein-Weyl equations (2.4) if and only if there exists a 2-parameter family of surfaces which are totally geodesic with respect to the connection $\mathbb{D}$ and null with respect to the conformal structure $[g]$. Such surfaces come from the associated dispersionless Lax pairs $[5,12,17]$.

2.4. Integrability via dispersionless Lax representation. A pair of Hamilton-Jacobi type equations for an auxiliary function $S$,

$$
\begin{aligned}
& S_{x_{2}}=f\left(S_{x_{1}}, u_{x_{i} x_{j}}\right), \\
& S_{x_{3}}=g\left(S_{x_{1}}, u_{x_{i} x_{j}}\right),
\end{aligned}
$$

is said to define a dispersionless Lax representation of equation (1.1) if (2.6) is compatible modulo (1.1). Lax pairs of this form appeared in [47] as dispersionless limits of Lax pairs of integrable soliton equations.

Example 2.3. Dispersionless Lax pair of the dKP equation (1.2) has the form

$$
\begin{aligned}
& S_{x_{2}}=\frac{1}{2} S_{x_{1}}^{2}+u_{x_{1} x_{1}}, \\
& S_{x_{3}}=\frac{1}{3} S_{x_{1}}^{3}+u_{x_{1} x_{1}} S_{x_{1}}+u_{x_{1} x_{2}} .
\end{aligned}
$$


Example 2.4. Dispersionless Lax pair of equation (1.4) has the form

$$
\begin{aligned}
& S_{x_{2}}=f\left(S_{x_{1}}, u_{x_{1} x_{1}}\right) u_{x_{1} x_{2}}+\frac{1}{3} g\left(S_{x_{1}}, u_{x_{1} x_{1}}\right) u_{x_{1} x_{3}}^{3}, \\
& S_{x_{3}}=f\left(S_{x_{1}}, u_{x_{1} x_{1}}\right) u_{x_{1} x_{3}} .
\end{aligned}
$$

Here the functions $f$ and $g$ are defined by (we set $S_{x_{1}}=x, u_{x_{1} x_{1}}=t$ ):

$$
g=f_{t}+f f_{x}, \quad f=-2(\ln v)_{x}
$$

where the function $v$ is the Jacobi theta series $\vartheta_{00}$, see (4.3) for the definition, evaluated at $(\tau, z)=\left(\frac{i t}{\pi}, \frac{x}{2 \pi}\right)$ :

$$
v(x, t)=\vartheta_{00}(i t / \pi, x / 2 \pi)=1+2 \sum_{n=1}^{\infty} e^{-n^{2} t} \cos (n x) .
$$

In some cases it is more convenient to deal with parametric Lax pairs,

$$
S_{x_{1}}=f\left(p, u_{x_{i} x_{j}}\right), \quad S_{x_{2}}=g\left(p, u_{x_{i} x_{j}}\right), \quad S_{x_{3}}=h\left(p, u_{x_{i} x_{j}}\right),
$$

where $p$ is an auxiliary parameter. Such Lax pairs were used in the construction of the universal Whitham hierarchy [30]. For example, parametric form of the dKP Lax pair (2.7) is

$$
S_{x_{1}}=p, \quad S_{x_{2}}=\frac{1}{2} p^{2}+u_{x_{1} x_{1}}, \quad S_{x_{3}}=\frac{1}{3} p^{3}+u_{x_{1} x_{1}} p+u_{x_{1} x_{2}} .
$$

Note that the compatibility condition of equations (2.8) is

$$
f_{p}\left(g_{x_{3}}-h_{x_{2}}\right)+g_{p}\left(h_{x_{1}}-f_{x_{3}}\right)+h_{p}\left(f_{x_{2}}-g_{x_{1}}\right)=0 .
$$

It follows from [5,17] that Hirota type equation (1.1) is integrable if and only if it has a dispersionless Lax representation (satisfying a suitable non-degeneracy condition).

\section{Hirota Master-Equation via the Odesskii-Sokolov Construction: Proof of Theorem 1.1}

It was proved in [36] that a generic integrable Hirota type equation (1.1) can be parametrised by generalised hypergeometric functions. Here we briefly summarise the construction. Consider the generalised hypergeometric system of Appell's type,

$$
\begin{aligned}
\frac{\partial^{2} h}{\partial v_{i} \partial v_{j}}= & \frac{s_{i}}{v_{i}-v_{j}} \frac{\partial h}{\partial v_{j}}+\frac{s_{j}}{v_{j}-v_{i}} \frac{\partial h}{\partial v_{i}}, \quad i \neq j, \\
\frac{\partial^{2} h}{\partial v_{i}^{2}}= & -\sigma \frac{s_{i}}{v_{i}\left(v_{i}-1\right)} h+\frac{s_{i}}{v_{i}\left(v_{i}-1\right)} \sum_{j \neq i}^{n} \frac{v_{j}\left(v_{j}-1\right)}{v_{j}-v_{i}} \frac{\partial h}{\partial v^{j}} \\
& +\left(\sum_{j \neq i}^{n} \frac{s_{j}}{v_{i}-v_{j}}+\frac{s_{i}+s_{n+1}}{v_{i}}+\frac{s_{i}+s_{n+2}}{v_{i}-1}\right) \frac{\partial h}{\partial v_{i}} .
\end{aligned}
$$

Here $s_{1}, \ldots, s_{n+2}$ are arbitrary constants (which we will assume to be rational), $\sigma=$ $1+s_{1}+\cdots+s_{n+2}$, and $h$ is a function of $n$ variables $v_{1}, \ldots, v_{n}$. This system is involutive and 
has $n+1$ linearly independent solutions known as generalised hypergeometric functions $[21,36]$. Introducing the differential

$$
\omega=t^{s_{n+1}}(t-1)^{s_{n+2}}\left(t-v_{1}\right)^{s_{1}} \ldots\left(t-v_{n}\right)^{s_{n}} d t,
$$

solutions to system (3.1) can be represented in terms of the corresponding periods, $\int_{p}^{q} \omega$ where $p, q \in\left\{0,1, \infty, v_{1}, \ldots, v_{n}\right\}$. This statement can be explicitly found in Mostow [33] who also noted that only $n+1$ of these periods are linearly independent [34], see also $[8,26]$. In low dimensions, analogous observations were made by Picard in 1883 [39]. With any generalised hypergeometric system (3.1) Odesskii and Sokolov associated a dispersionless integrable system in 3D possessing a dispersionless Lax representation [36].

We will need a particular case of the general construction with $n=5$ and $s_{4}=s_{5}=\frac{1}{2}$, $s_{1}=s_{2}=s_{3}=s_{6}=s_{7}=-\frac{1}{2}$, see example 5 of [36]. The first observation of [36] is that one can choose a basis of solutions $h_{1}, h_{2}, h_{3}, g_{1}, g_{2}, g_{3}$ of the corresponding system (3.1) such that

$$
\begin{aligned}
& \Delta\left(g_{1}, h_{3}, h_{1}\right)=\Delta\left(g_{2}, h_{2}, h_{3}\right), \\
& \Delta\left(g_{1}, h_{1}, h_{2}\right)=\Delta\left(g_{3}, h_{2}, h_{3}\right), \\
& \Delta\left(g_{2}, h_{1}, h_{2}\right)=\Delta\left(g_{3}, h_{3}, h_{1}\right),
\end{aligned}
$$

where we use the notation

$$
\Delta\left(f_{1}, f_{2}, f_{3}\right)=\operatorname{det}\left(\begin{array}{ccc}
f_{1} & f_{2} & f_{3} \\
f_{1, v_{4}} & f_{2, v_{4}} & f_{3, v_{4}} \\
f_{1, v_{5}} & f_{2, v_{5}} & f_{3, v_{5}}
\end{array}\right) .
$$

With these data Odesskii and Sokolov associated a dispersionless Hirota type equation (1.1) for an auxiliary function $\mathcal{U}$ depending on $x_{1}, x_{2}, x_{3}$ represented parametrically as

$$
\begin{array}{lll}
\frac{\Delta\left(g_{1}, h_{2}, h_{3}\right)}{\Delta}=\mathcal{U}_{x_{1} x_{1}}, & \frac{\Delta\left(g_{2}, h_{3}, h_{1}\right)}{\Delta}=\mathcal{U}_{x_{2} x_{2}}, & \frac{\Delta\left(g_{3}, h_{1}, h_{2}\right)}{\Delta}=\mathcal{U}_{x_{3} x_{3}}, \\
\frac{\Delta\left(g_{1}, h_{3}, h_{1}\right)}{\Delta}=\mathcal{U}_{x_{1} x_{2}}, & \frac{\Delta\left(g_{1}, h_{1}, h_{2}\right)}{\Delta}=\mathcal{U}_{x_{1} x_{3}}, & \frac{\Delta\left(g_{2}, h_{1}, h_{2}\right)}{\Delta}=\mathcal{U}_{x_{2} x_{3}},
\end{array}
$$

with $\Delta=\Delta\left(h_{1}, h_{2}, h_{3}\right)$. The required equation results on the elimination of the 5 parameters $v_{1}, \ldots, v_{5}$ from the 6 relations (3.3). This equation was shown to be integrable via a dispersionless Lax representation of type (2.8).

The geometry behind this construction is as follows. With the choice of constants $s_{i}$ specified above the differential $\omega$ takes the form

$$
\omega=\frac{\left(t-v_{4}\right)\left(t-v_{5}\right)}{v} d t, \quad v=\sqrt{t(t-1)\left(t-v_{1}\right) \ldots\left(t-v_{5}\right)},
$$

which is a holomorphic differential on the genus 3 hyperelliptic curve $\mathcal{C}$

$$
v^{2}=t(t-1)\left(t-v_{1}\right) \ldots\left(t-v_{5}\right) .
$$

Choosing a system of cycles $a_{i}, b_{j}$ on the curve $\mathcal{C}$ with the intersection matrix $a_{i} \cdot b_{j}=\delta_{i j}$ we denote by $h_{1}, h_{2}, h_{3}, g_{1}, g_{2}, g_{3}$ the corresponding periods of $\omega$ which form a basis of solutions of the associated system (3.1). We will see that this basis automatically satisfies constraints (3.2) due to the Riemann relations. The basis of holomorphic differentials on $\mathcal{C}$ is given by

$$
\omega=\frac{\left(t-v_{4}\right)\left(t-v_{5}\right)}{v} d t, \quad \frac{\partial \omega}{\partial v_{4}}=-\frac{1}{2} \frac{\left(t-v_{5}\right)}{v} d t, \quad \frac{\partial \omega}{\partial v_{5}}=-\frac{1}{2} \frac{\left(t-v_{4}\right)}{v} d t .
$$


Their periods over $a$ and $b$ cycles are given by the matrix

$$
\left(\begin{array}{cccccc}
h_{1} & h_{2} & h_{3} & g_{1} & g_{2} & g_{3} \\
h_{1, v_{4}} & h_{2, v_{4}} & h_{3, v_{4}} & g_{1, v_{4}} & g_{2, v_{4}} & g_{3, v_{4}} \\
h_{1, v_{5}} & h_{2, v_{5}} & h_{3, v_{5}} & g_{1, v_{5}} & g_{2, v_{5}} & g_{3, v_{5}}
\end{array}\right) .
$$

Multiplying this matrix from the left by the inverse of $\left(\begin{array}{ccc}h_{1} & h_{2} & h_{3} \\ h_{1, v_{4}} & h_{2, v_{4}} & h_{3, v_{4}} \\ h_{1, v_{5}} & h_{2, v_{5}} & h_{3, v_{5}}\end{array}\right)$ we get

$$
\left(\begin{array}{llllll}
1 & 0 & 0 & \tau_{11} & \tau_{12} & \tau_{13} \\
0 & 1 & 0 & \tau_{21} & \tau_{22} & \tau_{23} \\
0 & 0 & 1 & \tau_{31} & \tau_{32} & \tau_{33}
\end{array}\right)=\left(I_{3} \tau\right)
$$

where $\tau$ is the period matrix of the curve $\mathcal{C}$. Explicitly we have

$$
\tau=\frac{1}{\Delta}\left(\begin{array}{c}
\Delta\left(g_{1}, h_{2}, h_{3}\right) \Delta\left(g_{2}, h_{2}, h_{3}\right) \Delta\left(g_{3}, h_{2}, h_{3}\right) \\
\Delta\left(g_{1}, h_{3}, h_{1}\right) \Delta\left(g_{2}, h_{3}, h_{1}\right) \Delta\left(g_{3}, h_{3}, h_{1}\right) \\
\Delta\left(g_{1}, h_{1}, h_{2}\right) \Delta\left(g_{2}, h_{1}, h_{2}\right) \Delta\left(g_{3}, h_{1}, h_{2}\right)
\end{array}\right) .
$$

The symmetry of the period matrix $\tau$ is equivalent to constraints (3.2). Bases satisfying (3.2) (such that matrix (3.4) has positive definite imaginary part) are thus in one-to-one correspondence with canonical systems of cycles on $\mathcal{C}$. In particular, all of them are $\operatorname{Sp}(6, \mathbb{R})$-related. Finally, relations (3.3) take the form $\tau=\operatorname{Hess}(\mathcal{U})$. The elimination of the parameters $v_{i}$ yields a Hirota type equation for $\mathcal{U}$ which by construction is the equation of the genus 3 hyperelliptic divisor,

$$
\vartheta_{m}(\tau)=\vartheta_{m}(\operatorname{Hess}(\mathcal{U}))=0
$$

where $\vartheta_{m}$ is any even theta constant, a result that goes back to Schottky, 1880, see e.g. [40]. Although there are 36 even theta constants, the corresponding equations are all equivalent due to the transitivity of the $\operatorname{Sp}(6, \mathbb{Z})$-action on the set of even theta characteristics. Note that if all parameters (branch points) $v_{i}$ of the hyperelliptic curve are real we can choose a basis of cycles such that the corresponding period matrix $\tau$ will be purely imaginary. Then $\mathcal{U}=i u$ will also be purely imaginary, and the Hirota equation

$$
\vartheta_{m}(i \operatorname{Hess}(\mathrm{u}))=0
$$

will be real.

The uniqueness (over $\mathbb{C}$ ) of the Hirota master-equation can be established as follows. Up to a suitable equivalence transformation, every equation (1.1) can be brought to a form where $\frac{\partial F}{\partial u_{x_{1} x_{1}}} \neq 0$. Thus, without any loss of generality, we can work with the evolutionary equation (2.2). The integrability conditions derived in Section 7 of [14] express all third-order partial derivatives of $f$ as explicit rational functions of its firstorder and second-order partial derivatives, symbolically,

$$
d^{3} f=P\left(d f, d^{2} f\right) .
$$

Equations (3.5) define a rational connected 21-dimensional affine variety $X$ in the affine space of third-order jets of $f$. The algebraic equivalence group $\operatorname{Sp}(6, \mathbb{C})$ (of dimension 21 ) acts on $X$ in a locally free way (see [14], note that local freeness holds over $\mathbb{C}$ as well), and therefore possesses a Zariski open orbit. The uniqueness of this orbit follows from the fact that any two Zariski open sets on a connected variety must necessarily intersect. 


\section{Examples}

In this section, we give a few more examples of integrable (non-generic) equations (1.1) with non-trivial modular properties.

4.1. Equations of the form $u_{x_{1} x_{2}}+u_{x_{1} x_{3}} f\left(u_{x_{2} x_{3}}, u_{x_{3} x_{3}}\right)=0$. This example has already been investigated $[2,3,15]$. For the reader's convenience, we explain it again. In this case, the integrability conditions obtained via the method of hydrodynamic reductions lead to the following system of third-order PDEs, we set $b=u_{x_{2} x_{3}}$ and $c=u_{x_{3} x_{3}}$ :

$$
\begin{aligned}
\left(f f_{b}-f_{c}\right) f_{b b b}= & f_{b b}\left(f f_{b b}+f_{b}^{2}-f_{b c}\right)=f_{b b}\left(f f_{b}-f_{c}\right)_{b}, \\
\left(f f_{b}-f_{c}\right) f_{b b c}= & f_{b b}\left(f f_{b c}+f_{b} f_{c}-f_{c c}\right)=f_{b b}\left(f f_{b}-f_{c}\right)_{c}, \\
\left(f f_{b}-f_{c}\right) f_{b c c}= & 2 f f_{b c}^{2}-f f_{b b} f_{c c}-f_{b c} f_{c c}+f_{b}^{2} f_{c c}-2 f_{b} f_{c} f_{b c}+2 f_{c}^{2} f_{b b}, \\
\left(f f_{b}-f_{c}\right) f_{c c c}= & 2 f^{2} f_{b c}^{2}-2 f^{2} f_{b b} f_{c c}+f f_{b c} f_{c c}+4 f f_{b}^{2} f_{c c} \\
& -8 f f_{b} f_{c} f_{b c}+4 f f_{c}^{2} f_{b b}-f_{c c}^{2}-f_{b} f_{c} f_{c c}+2 f_{c}^{2} f_{b c} .
\end{aligned}
$$

We will show that a generic solution of this system is given by the logarithmic derivative of any Jacobi theta series with characteristics.

The first two equations lead to the Burgers' equation $f f_{b}-f_{c}=v f_{b b}$ where $v \in \mathbb{C}^{*}$. Without any loss of generality, we set $v=-1$ and using classical results about Burgers' equation, we can write the function $f$ as $f=2 \frac{\psi_{b}}{\psi}$ where the function $\psi$ satisfies the heat equation: $\psi_{c}=\psi_{b b}$. By substituting $f=2 \frac{\psi_{b}}{\psi}$ in the equations (4.1) and reducing modulo $\psi_{c}=\psi_{b b}$, the first two equations are automatically satisfied while the third one gives the following sixth-order ordinary differential equation $\left(\psi^{(i)}\right.$ stands for $\left.\frac{\partial^{i} \psi}{\partial b^{i}}\right)$

$$
\begin{aligned}
& \left(\psi^{\prime \prime \prime} \psi^{(6)}-\psi^{(4)} \psi^{(5)}\right) \psi^{4}+\left(2 \psi^{\prime \prime \prime}\left(\left(\psi^{\prime \prime \prime}\right)^{2}-\psi^{\prime} \psi^{(5)}\right)+3 \psi^{\prime \prime}\left(\psi^{\prime \prime} \psi^{(5)}-\psi^{\prime} \psi^{(6)}\right)\right. \\
& \left.\quad+5 \psi^{(4)}\left(\psi^{\prime} \psi^{(4)}-\psi^{\prime \prime} \psi^{\prime \prime \prime}\right)\right) \psi^{3}+\left(2\left(\psi^{\prime}\right)^{3} \psi^{(6)}+6\left(\psi^{\prime}\right)^{2} \psi^{\prime \prime} \psi^{(5)}-10\left(\psi^{\prime}\right)^{2} \psi^{\prime \prime \prime} \psi_{(4.2)}^{(4)}\right. \\
& \left.\quad+2 \psi^{\prime} \psi^{\prime \prime} \psi^{\prime \prime \prime 2}\right) \psi^{2}+6\left(2\left(\psi^{\prime}\right)^{3} \psi^{\prime \prime \prime 2}-\left(\psi^{\prime}\right)^{4} \psi^{(5)}-\left(\psi^{\prime} \psi^{\prime \prime}\right)^{2} \psi^{\prime \prime \prime}\right) \psi \\
& \quad+6\left(\psi^{\prime}\right)^{3}\left(\left(\psi^{\prime}\right)^{2} \psi^{(4)}-2 \psi^{\prime} \psi^{\prime \prime} \psi^{\prime \prime \prime}+\left(\psi^{\prime \prime}\right)^{3}\right)=0 .
\end{aligned}
$$

Finally the last equation of (4.1) gives the derivative with respect to $b$ of the latter one. We introduce the following Jacobi theta series with characteristics:

$$
\vartheta_{\alpha \beta}(\tau, z)=\sum_{n \in \mathbb{Z}} e^{\pi i\left((n+\alpha / 2)^{2} \tau+2(n+\alpha / 2)(z+\beta / 2)\right)}
$$

where $\tau \in \mathfrak{H}_{1}=\{\tau \in \mathbb{C} \mid \operatorname{Im}(\tau)>0\}, z \in \mathbb{C}$ and $(\alpha, \beta) \in \mathbb{R}^{2}$ (characteristics). Recall that each Jacobi theta function satisfies the following PDE (heat equation):

$$
4 i \pi \frac{\partial \vartheta_{\alpha \beta}}{\partial \tau}=\frac{\partial^{2} \vartheta_{\alpha \beta}}{\partial z^{2}} .
$$

So the function $\psi(b, c)=\vartheta_{\alpha \beta}\left(\frac{i c}{\pi}, \frac{b}{2 \pi}\right)$ satisfies the equation $\psi_{c}=\psi_{b b}$. It remains to check that such a function $\psi$ satisfies the equation (4.2). Note that if $h$ is a solution of (4.2) then the following two expressions

$$
f_{1}(b, c)=h\left(b+g_{1}(c), c\right), \quad f_{2}(b, c)=g_{2}(c) e^{b g_{3}(c)} h(b, c)
$$


are also solutions of (4.2) for any functions $g_{i}$; for $f_{1}$ the proof is obvious while for $f_{2}$ it is a short computation. Using the formula

$$
\psi(b, c)=\vartheta_{\alpha \beta}\left(\frac{i c}{\pi}, \frac{b}{2 \pi}\right)=e^{\frac{\pi i}{2} \alpha \beta} e^{-c \frac{\alpha^{2}}{4}} e^{\frac{i \alpha}{2} b} \vartheta_{00}\left(\frac{i c}{\pi}, \frac{b}{2 \pi}+\frac{i c}{\pi} \frac{\alpha}{2}+\frac{\beta}{2}\right),
$$

it is therefore sufficient to prove that the function $\vartheta_{00}$ evaluated at $(\tau, z)=\left(\frac{i c}{\pi}, \frac{b}{2 \pi}\right)$ satisfies the ODE (4.2). The differential operator defined by the left hand side of (4.2), say $\mathcal{D}$, has the following two properties: for $\left(\begin{array}{ll}\alpha & \beta \\ \gamma & \delta\end{array}\right)$ in a suitable subgroup of $\operatorname{SL}(2, \mathbb{R})$ and $(\lambda, \mu)$ in a suitable sublattice of $\mathbb{R}^{2}$, assume that a function $h$ transforms as follows

$h\left(\frac{b}{\gamma c+\delta}, \frac{\alpha c+\beta}{\gamma c+\delta}\right)=(\gamma c+\delta)^{\frac{1}{2}} e^{\frac{\gamma b^{2}}{\gamma c+\delta}} h(b, c), \quad h(b+\lambda c+\mu, c)=e^{\left(\lambda^{2} c+2 \lambda b\right)} h(b, c)$

then

$$
\begin{gathered}
\mathcal{D} h\left(\frac{b}{\gamma c+\delta}, \frac{\alpha c+\beta}{\gamma c+\delta}\right)=(\gamma c+\delta)^{12} e^{6 \frac{\gamma b^{2}}{\gamma c+\delta}} \mathcal{D} h(b, c), \\
\mathcal{D} h(b+\lambda c+\mu, c)=e^{6\left(\lambda^{2} c+2 \lambda b\right)} \mathcal{D} h(b, c) .
\end{gathered}
$$

This indicates that the operator $\mathcal{D}$ sends, up to the condition on the Fourier expansion, Jacobi forms of weight one-half and index one-half to Jacobi forms of weight 12 and index 3 on a suitable subgroup of $\operatorname{SL}(2, \mathbb{R})$. Recalling that the Jacobi theta series $\vartheta_{00}$ can be viewed as a Jacobi form of weight one-half and index one-half (with a multiplier system) on the principal congruence subgroup of level 4, checking that the function $\vartheta_{00}$ evaluated at $(\tau, z)=\left(\frac{i c}{\pi}, \frac{b}{2 \pi}\right)$ satisfies the ODE (4.2) reduces to check it for sufficiently many Fourier coefficients (finite dimension of spaces of Jacobi forms) which can be done by any computer algebra systems. We conclude that any Jacobi theta series evaluated at $(\tau, z)=\left(\frac{i c}{\pi}, \frac{b}{2 \pi}\right)$ satisfies the ODE (4.2) and the PDE $\psi_{c}=\psi_{b b}$. Taking for example $\psi(b, c)=\vartheta_{00}\left(\frac{i c}{\pi}, \frac{b}{2 \pi}\right)$, we get for the function $f$

$$
\begin{aligned}
f(b, c)= & 2 \frac{\psi_{b}}{\psi}(b, c)=-4\left(\sin (b) e^{-c}-\sin (2 b) e^{-2 c}\right. \\
& \left.+(\sin (b)+\sin (3 b)) e^{-3 c}-\sin (4 b) e^{-4 c}+\cdots\right) .
\end{aligned}
$$

4.2. Equations of the form $u_{x_{1} x_{2}}+u_{x_{1} x_{3}} u_{x_{2} x_{3}} r\left(u_{x_{3} x_{3}}\right)=0$. This case is obtained as a specialisation of the previous one:

$$
f\left(u_{x_{2} x_{3}}, u_{x_{3} x_{3}}\right)=u_{x_{2} x_{3}} r\left(u_{x_{3} x_{3}}\right) .
$$

The integrability conditions in this case are obtained by substituting $f(b, c)=b r(c)$ in the system (4.1). This leads to a single third-order ordinary differential equation for the function $r$, we set $r^{(k)}=\frac{d^{k} r}{d c^{k}}$ :

$$
r^{\prime \prime \prime}\left(r^{\prime}-r^{2}\right)-\left(r^{\prime \prime}\right)^{2}+4 r^{3} r^{\prime \prime}+2\left(r^{\prime}\right)^{3}-6\left(r r^{\prime}\right)^{2}=0 .
$$

This case has already been investigated in Section 4.5 of [3]. Let us briefly recall the results obtained there: we denote by $\operatorname{Dr}(c)$ the left hand side of (4.5). As noticed in [3], 
the expression $\mathcal{D} r(c)$ has a $\operatorname{SL}(2, \mathbb{R})$-invariance: for any $\left(\begin{array}{ll}\alpha & \beta \\ \gamma & \delta\end{array}\right) \in \operatorname{SL}(2, \mathbb{R})$, assume that the function $r$ satisfies the following functional equation

$$
\tilde{r}(c)=r\left(\frac{\alpha c+\beta}{\gamma c+\delta}\right)=(\gamma c+\delta)^{2} r(c)+\gamma(\gamma c+\delta)
$$

then we have

$$
\mathcal{D} \tilde{r}(c)=\mathcal{D} r\left(\frac{\alpha c+\beta}{\gamma c+\delta}\right)=(\gamma c+\delta)^{12} \mathcal{D} r(c) .
$$

Then modulo the $\operatorname{SL}(2, \mathbb{R})$-action given by (4.6), the generic solution of (4.5) is given by $(c<0)$ :

$$
r(c)=1-8 \sum_{n \geq 1} \sigma_{1}^{-}(n) e^{4 n c} \text { with } \sigma_{1}^{-}(n)=\sum_{d \mid n}(-1)^{d} d .
$$

Note that for $\tau \in \mathfrak{H}_{1}, r\left(\frac{\pi i \tau}{2}\right)=1-8 \sum_{n \geq 1} \sigma_{1}^{-}(n) e^{2 \pi i n \tau}$ is a quasi-modular form of weight 2 on the congruence subgroup $\Gamma_{0}(2)$. In fact we have $r\left(\frac{\pi i \tau}{2}\right)=\left(4 e_{2}(2 \tau)-\right.$ $\left.e_{2}(\tau)\right) / 3$ where $e_{2}$ is the Eisenstein series of weight 2 on $\operatorname{SL}(2, \mathbb{Z})$, see (1.5).

We would like to give another description of the generic solution which will make a connection with the Legendre family of elliptic curves. We make the change of functions $r=g^{\prime} / g$ in (4.5), then the function $g$ satisfies the following fourth-order ordinary differential equation:

$$
\begin{gathered}
g^{\prime \prime \prime \prime \prime}\left(g^{\prime \prime} g-2 g^{\prime 2}\right) g^{3}-g^{\prime \prime \prime 2} g^{4}+2 g^{2} g^{\prime} g^{\prime \prime \prime}\left(4 g^{2}+g^{\prime \prime} g\right) \\
-g^{\prime \prime 3} g^{3}-3 g^{\prime \prime 2} g^{\prime 2} g^{2}-12 g^{\prime \prime} g^{\prime 4} g+8 g^{\prime 6}=0 .
\end{gathered}
$$

Section 5 of [7] proposed a method for linearising any equation of the form (4.5) which has been carried out explicitly along the proof of theorem 3 of [18]. This gives the following hypergeometric differential equation associated to the equation (4.5):

$$
s(1-s) w^{\prime \prime}(s)+(1-2 s) w^{\prime}(s)-\frac{1}{4} w(s)=0 .
$$

Let $w_{1}$ and $w_{2}$ be two linearly independent solutions of this hypergeometric equation with the Wronskian normalised as $w_{2} w_{1, s}-w_{1} w_{2, s}=\frac{1}{s(1-s)}$. These solutions can be written as periods of the holomorphic differential 1-form $\omega$ given by

$$
\omega=\frac{d t}{\sqrt{t(t-1)(t-s)}}
$$

of the elliptic curve $v^{2}=t(t-1)(t-s)$, we assume $s \in(0,1)$. Let us choose a basis of solutions such that $w_{1}$ has a logarithmic singularity at zero while $w_{2}$ is the standard hypergeometric series with parameters $(1 / 2,1 / 2 ; 1)$ :

$$
\begin{aligned}
& w_{1}(s)=-i \int_{-\infty}^{0} \omega+\frac{4 \ln 2}{\pi} \int_{1}^{\infty} \omega=w_{2}(s) \ln s+2 \sum_{n=1}^{\infty}\left(\frac{(2 n-1) ! !}{2^{n} n !}\right)^{2}\left(\sum_{k=1}^{n} \frac{1}{k(2 k-1)}\right) s^{n}, \\
& w_{2}(s)=\frac{1}{\pi} \int_{1}^{\infty} \omega=F\left(\frac{1}{2}, \frac{1}{2} ; 1 ; s\right) .
\end{aligned}
$$


This choice satisfies the Wronskian constraint. Noticing that equation (4.7) has the following $\operatorname{SL}(2, \mathbb{R})$-symmetry which comes directly from (4.6):

$$
\tilde{c}=\frac{\alpha c+\beta}{\gamma c+\delta}, \quad \tilde{g}=(\gamma c+\delta) g
$$

we obtain a generic solution via parametric formulae (viewing $s$ as a parameter)

$$
c=\frac{w_{1}(s)}{w_{2}(s)} \text { and } g=w_{2}(s) .
$$

It is a classical fact that the first relation can be inverted and this gives $s(c)=\frac{\vartheta_{10}^{4}(i c, 0)}{\vartheta_{00}^{4}(i c, 0)}$ and the substitution into $g=w_{2}$ gives

$$
g(c)=w_{2}(s(c))=F\left(\frac{1}{2}, \frac{1}{2} ; 1 ; s(c)\right)=F\left(\frac{1}{2}, \frac{1}{2} ; 1 ; \frac{\vartheta_{10}^{4}(i c, 0)}{\vartheta_{00}^{4}(i c, 0)}\right)=\vartheta_{00}^{2}(i c, 0) .
$$

We refer to $[4,27]$ for a review of these classical formulae.

Remark 4.1. Since the action of the group $\operatorname{SL}(2, \mathbb{Z})$ permutes the Jacobi theta series with characteristics in $\{0,1\}$ and the equation (4.7) admits a $\operatorname{SL}(2, \mathbb{R})$-symmetry, another solution to the equation (4.7) can be chosen as $g(c)=\vartheta_{10}^{2}(i c, 0)$. For $\tau \in \mathfrak{H}_{1}$, let $f(\tau)=\vartheta_{10}^{2}(\tau, 0)$. It is a well-known fact that the function $f$ is modular form of weight 1 on $\Gamma_{0}(2)$ with a character. A direct computation shows that the logarithmic derivative of $f$ transforms like a quasi-modular form of weight 2 on $\Gamma_{0}(2)$, note the cancellation of the character, and we have

$$
\frac{f^{\prime}}{f}(\tau)=2 \frac{\vartheta_{10}^{\prime}(\tau, 0)}{\vartheta_{10}(\tau, 0)}=\frac{\pi i}{2} r\left(\frac{\pi i \tau}{2}\right) .
$$

The latter formula connects the two descriptions presented in this section.

\section{Concluding Remarks}

The attempts to generalise/specialise Theorem 1.1 lead to the following observations:

- Some compactifications of the hyperelliptic divisor in genus 3 [19] should be related to the classification of special integrable Hirota type equations (1.1) that have continuous symmetries from the equivalence group $\operatorname{Sp}(6, \mathbb{R})$. Such degenerations could be interesting from the point of view of their potential applications.

- The construction of Odesskii and Sokolov [36] can be adapted to show that for any higher genus $g \geq 4$ the hyperelliptic locus defines a 3D integrable hierarchy of Hirota-type equations (in the notation of [36] this corresponds to the choice of constants $s_{1}=\cdots=s_{g}=s_{2 g}=s_{2 g+1}=\frac{1}{2}, s_{g+1}=s_{2 g-1}=-\frac{1}{2}, n=2 g-$ $1, k=g$ ). Here the equations of the hierarchy coincide with the defining equations of the hyperelliptic locus $[24,35,40,41]$, which is known to be characterised by the vanishing of $\frac{1}{2}(g-1)(g-2)$ theta constants (Hirota equations result on the substitution of $\tau=i \operatorname{Hess}(u)$ for the $g \times g$ period matrix). 
- Although it would be tempting to conjecture that the Schottky divisor (image of the Torelli map $\mathcal{M}_{4} \hookrightarrow \mathcal{A}_{4}$ from the moduli space of curves of genus 4 to the moduli space of principally polarised Abelian varieties [9]) corresponds to an integrable Hirota type equation in $4 \mathrm{D}$, this is no longer the case. Recent results of [16] show that in 4D the requirement of integrablity implies the symplectic Monge-Ampère property, which leads to a complete list of integrable heavenly-type equations classified in [10]. Thus, the occurrence of modular forms and theta functions in the classification of integrable Hirota type equations is the essentially 3-dimensional phenomenon.

- Integrable symplectic Monge-Ampère equations in 4D correspond to hypersurfaces $M^{9}$ in the Lagrangian Grassmannian $\Lambda^{10}$ which can be characterised as special hyperplane sections of the Plücker embedding $\Lambda^{10} \subset \mathbb{P}^{41}$ (see [10]). In 3D we have a similar situation: embedding the quotient of the Siegel upper half space $\mathfrak{H}_{3}$ by the group $\Gamma_{3}(4,8)$ into $\mathbb{P}^{35}$ via the 36 even theta constants we obtain a projective variety cut out by quartics (see [23]). In this picture, hyperelliptic divisor is cut out by the coordinate hyperplanes in $\mathbb{P}^{35}$.

- Finally, it would be interesting to obtain a purely computational proof of Theorem 1.1 by directly proving that even theta constants satisfy the integrability conditions obtained by the method of hydrodynamic reductions, see Sect. 2.1.

Acknowledgements. We thank B. Dubrovin, I. Krichever, M. Pavlov, A. Prendergast-Smith, and A. Veselov for clarifying discussions. We also thank the referees for useful suggestions. This research was supported by the EPSRC Grant EP/N031369/1.

Open Access This article is distributed under the terms of the Creative Commons Attribution 4.0 International License (http://creativecommons.org/licenses/by/4.0/), which permits unrestricted use, distribution, and reproduction in any medium, provided you give appropriate credit to the original author(s) and the source, provide a link to the Creative Commons license, and indicate if changes were made.

Publisher's Note Springer Nature remains neutral with regard to jurisdictional claims in published maps and institutional affiliations.

\section{References}

1. Boyer, C.P., Finley, J.D.: Killing vectors in self-dual Euclidean Einstein spaces. J. Math. Phys. 23, 1126$1130(1982)$

2. Buchstaber, V.M., Leikin, D.V., Pavlov, M.V.: The Egorov hydrodynamic chains, the Chazy equation and SL $(2, \mathbb{C})$ group. Funct. Anal. Appl. 37, 13-26 (2003)

3. Burovskiy, P.A., Ferapontov, E.V., Tsarev, S.P.: Second order quasilinear PDEs and conformal structures in projective space. Int. J. Math. 21(6), 799-841 (2010)

4. Bruinier, J., van der Geer, G., Harder, G., Zagier, D.: The 1-2-3 of Modular Forms. Universitext. Springer, Berlin (2008)

5. Calderbank, D.M.J., Kruglikov, B.: Integrability via geometry: dispersionless differential equations in three and four dimensions. arXiv:1612.02753

6. Cartan, É.: Sur une classe d'espaces de Weyl. Ann. Sci. École Norm. Sup. (3) 60, 1-16 (1943)

7. Clarkson, P.A., Olver, P.J.: Symmetry and the Chazy equation. J. Differ. Equ. 124(1), 225-246 (1996)

8. Deligne, P., Mostow, G.D.: Monodromy of hypergeometric functions and nonlattice integral monodromy. Inst. Hautes Études Sci. Publ. Math. 63, 5-89 (1986)

9. Donagi, R.: The Schottky problem, Theory of moduli (Montecatini Terme, 1985), Lecture Notes in Math., 1337, pp. 84-137, Springer, Berlin (1988)

10. Doubrov, B., Ferapontov, E.V.: On the integrability of symplectic Monge-Ampère equations. J. Geom. Phys. 60, 1604-1616 (2010)

11. Dubrovin, B.A., Novikov, S.P.: Hydrodynamics of weakly deformed soliton lattices: differential geometry and Hamiltonian theory. Russ. Math. Surv. 44(6), 35-124 (1989)

12. Dunajski, M., Mason, L.J., Tod, P.: Einstein-Weyl geometry, the dKP equation and twistor theory. J. Geom. Phys. 37(1-2), 63-93 (2001) 
13. Ferapontov, E.V., Khusnutdinova, K.R.: On the integrability of $(2+1)$-dimensional quasilinear systems. Commun. Math. Phys. 248, 187-206 (2004)

14. Ferapontov, E.V., Hadjikos, L., Khusnutdinova, K.R.: Integrable equations of the dispersionless Hirota type and hypersurfaces in the Lagrangian Grassmannian. Int. Math. Res. Not. 3, 496-535 (2010)

15. Ferapontov, E.V., Khusnutdinova, K.R., Pavlov, M.V.: Classification of integrable $(2+1)$-dimensional quasilinear hierarchies. Theor. Math. Phys. 144, 35-43 (2005)

16. Ferapontov, E.V., Kruglikov, B., Novikov, V.S.: Integrability of dispersionless Hirota type equations in 4D and the symplectic Monge-Ampère property. arXiv:1707.08070

17. Ferapontov, E.V., Kruglikov, B.S.: Dispersionless integrable systems in 3D and Einstein-Weyl geometry. J. Differ. Geom. 97, 215-254 (2014)

18. Ferapontov, E., Odesskii, A.V.: Integrable Lagrangians and modular forms. J. Geom. Phys. 60(6-8), 896-906 (2010)

19. Freitag, E., Manni, R.: Salvati: the modular variety of hyperelliptic curves of genus three. Trans. Am. Math. Soc. 363(1), 281-312 (2011)

20. Gauss, C.F.: Mathematisches Tagebuch 17961814, Ostwalds Klassiker der exakten Wissenschaften, 256. Geest und Portig, Leipzig (1976)

21. Gelfand, I.M., Graev, M.I., Retakh, V.S.: General hypergeometric systems of equations and series of hypergeometric type. Russ. Math. Surv. 47(4), 1-88 (1992)

22. Gibbons, J., Tsarev, S.P.: Reductions of the Benney equations. Phys. Lett. A 211, 19-24 (1996)

23. Glass, J.P.: Theta constants of genus three. Compos. Math. 40(1), 123-137 (1980)

24. Grushevsky, S.: Cubic equations for the hyperelliptic locus. Asian J. Math. 8(1), 161-172 (2004)

25. Hitchin, N.J.: Complex manifolds and Einstein's equations, Twistor geometry and nonlinear systems (Primorsko, 1980), Lecture Notes in Math., vol. 970, pp. 73-99. Springer, Berlin (1982)

26. Holzapfel, R.-P.: Geometry and arithmetic. Around Euler partial differential equations. Mathematische Monographien [Mathematical Monographs], vol. 20, p. 184. VEB Deutscher Verlag der Wissenschaften, Berlin (1986). ISBN: 3-326-00013-8

27. Husemöller, D.: Elliptic Curves. Springer, Berlin (2004)

28. Igusa, J.-I.: Modular forms and projective invariants. Am. J. Math. 89, 817-855 (1967)

29. Igusa, J.-I.: Theta Functions, Die Grundlehren der mathematischen Wissenschaften, vol. 194. Springer, Berlin (1972)

30. Krichever, I.M.: The $\tau$-function of the universal Whitham hierarchy, matrix models and topological field theories. Commun. Pure Appl. Math. 47(4), 437-475 (1994)

31. Krichever, I.M., Marshakov, A., Zabrodin, A.: Integrable structure of the Dirichlet boundary problem in multiply-connected domains. Commun. Math. Phys. 259(1), 1-44 (2005)

32. Marshakov, A., Wiegmann, P., Zabrodin, A.: Integrable structure of the Dirichlet boundary problem in two dimensions. Commun. Math. Phys. 227, 131-153 (2002)

33. Mostow, G.D.: Generalized Picard lattices arising from half-integral conditions. Inst. Hautes Études Sci. Publ. Math. 63, 91-106 (1986)

34. Mostow, G.D.: Existence of nonarithmetic monodromy groups. Proc. Nat. Acad. Sci. USA 78(10), 59485950 (1981)

35. Mumford, D.: Tata Lectures on Theta, 2, Progr. Math., 43. Birkhäuser, Boston (1984)

36. Odesskii, A.V., Sokolov, V.V.: Integrable pseudopotentials related to generalized hypergeometric functions. Sel. Math. 16, 145 (2010)

37. Pavlov, M.V.: New integrable (2+1)-equations of hydrodynamic type. Russ. Math. Surv. 58(2), 384-385 (2003)

38. Pavlov, M.V.: Classifying integrable Egoroff hydrodynamic chains. Theor. Math. Phys. 138, 45-58 (2004)

39. Picard, É.: Sur des fonctions de deux variables indépendantes analogues aux fonctions modulaires. Acta Math. 2, 114-135 (1883)

40. Poor, C.: The hyperelliptic locus. Duke Math. J. 76(3), 809-884 (1994)

41. Salvati Manni, R.: Modular forms vanishing on the hyperelliptic locus. Jpn. J. Math. (N.S.) 29(1), 135-142 (2003)

42. Smith, A.D.: Integrable GL(2) geometry and hydrodynamic partial differential equations. Commun. Anal. Geom. 18(4), 743-790 (2010)

43. Tsarev, S.P.: The geometry of Hamiltonian systems of hydrodynamic type. The generalized hodograph method. Math. USSR Izvest. 37, 397-419 (1991)

44. Wiegmann, P.B., Zabrodin, A.: Conformal maps and integrable hierarchies. Commun. Math. Phys. 213(3), 523-538 (2000)

45. Zabolotskaya, E.A., Khokhlov, R.V.: Quasi-plane waves in the nonlinear acoustics of confined beams. Sov. Phys. Acoust. 15, 35-40 (1969)

46. Zabrodin, A.V.: The dispersionless limit of the Hirota equations in some problems of complex analysis. Theor. Math. Phys. 129(2), 1511-1525 (2001) 
47. Zakharov, E.V.: Dispersionless limit of integrable systems in $2+1$ dimensions. In: Ercolani, N.M., et al. (eds.) Singular Limits of Dispersive Waves, pp. 165-174. Plenum Press, New York (1994)

Communicated by N. Nekrasov 